\title{
Applications of Cesàro Submethod to Trigonometric Approximation of Signals (Functions) Belonging to Class $\operatorname{Lip}(\alpha, p)$ in $L_{p}$-Norm
}

\author{
M. L. Mittal and Mradul Veer Singh \\ Department of Mathematics, Indian Institute of Technology Roorkee, Roorkee 247667, India \\ Correspondence should be addressed to Mradul Veer Singh; mradul.singh@gmail.com \\ Received 28 July 2015; Revised 18 November 2015; Accepted 11 February 2016 \\ Academic Editor: Mohsen Tadi
}

Copyright (C) 2016 M. L. Mittal and M. V. Singh. This is an open access article distributed under the Creative Commons Attribution License, which permits unrestricted use, distribution, and reproduction in any medium, provided the original work is properly cited.

We prove two Theorems on approximation of functions belonging to Lipschitz class $\operatorname{Lip}(\alpha, p)$ in $L_{p}$-norm using Cesàro submethod. Further we discuss few corollaries of our Theorems and compare them with the existing results. We also note that our results give sharper estimates than the estimates in some of the known results.

Dedicated to Professor S. C. Gupta

\section{Introduction}

For a given signal (function) $f \in L_{p}:=L_{p}[0,2 \pi], p \geq 1$, let

$$
\begin{aligned}
s_{n}(f) & :=s_{n}(f ; x)=\frac{a_{0}}{2}+\sum_{k=1}^{n}\left(a_{k} \cos k x+b_{k} \sin k x\right) \\
& =\sum_{k=0}^{n} u_{k}(f ; x)
\end{aligned}
$$

denote the partial sums, called trigonometric polynomials of degree (or order) $n$, of the first $(n+1)$ terms of the Fourier series of $f$ at a point $x$.

Define

$$
\tau_{n}(f)=\tau_{n}(f ; x)=\sum_{k=0}^{n} a_{n, k} s_{k}(f), \quad \forall n \geq 0 .
$$

The trigonometric Fourier series of signal $f$ is said to be $T$ summable to $s$, if $\tau_{n}(f) \rightarrow s$ as $n \rightarrow \infty$.

Throughout $T \equiv\left(a_{n, k}\right)$, a linear operator, will denote an infinite lower triangular matrix with nonnegative entries and row sums 1. Such a matrix $T$ is said to have monotone rows if, $\forall n,\left\{a_{n, k}\right\}$ is either nonincreasing or nondecreasing in $k$, $0 \leq k \leq n$. A linear operator $T$ is said to be regular if it is limitpreserving over the space of convergent sequences.

Note 1. In (2), $T \equiv\left(a_{n, k}\right)$ behaves as a digital filter if $s_{k}$ and $\tau_{n}$ denote the input and output sequences of information, respectively. By taking different values of $a_{n, k}$, we get a variety of digital filters, For example, if $a_{n, k}=(n+1)^{-1}$, we get a very popular digital filter known as Cesàro filter. Psarakis and Moustakides [1, p. 591] have mentioned that the digital filters are widely used in various signal processing applications. During past few decades, various techniques for designing digital filters have been suggested. It is worth mentioning that the most common techniques use, as approximation criterion, the minimization of the $L_{p}$-measure $1 \leq p \leq \infty$. Thus the designing of digital filters has been recognized as an approximation problem. Here we are interested in approximations of functions in $L_{1}$-space.

A signal (function) $f$ in $L_{p}$ is approximated by trigonometric polynomials $\tau_{n}$ of order (or degree) $n$ and the degree 
of approximation $E_{n}(f)$ is given by $E_{n}(f)=\min _{n} \| f(x)-$ $\tau_{n}(f ; x) \|_{p}$. This method of approximation is called trigonometric Fourier approximation (tfa). Further nice applications of tfa can be found in $[2,3]$ and the references given there.

The integral modulus of continuity [4] of $f$ is defined by

$$
\begin{aligned}
& \omega_{p}(\delta ; f) \\
& \quad:=\sup _{0<|h| \leq \delta}\left\{\frac{1}{2 \pi} \int_{0}^{2 \pi}|f(x+h)-f(x)|^{p} d x\right\}^{1 / p} .
\end{aligned}
$$

If, for $\alpha>0, \omega_{p}(\delta ; f)=O\left(\delta^{\alpha}\right)$, then $f \in \operatorname{Lip}(\alpha, p)(p \geq 1)$. Throughout $\|\cdot\|_{p}$ will denote the $L_{p}$-norm, defined by $\|f\|_{p}:=$ $\left\{(1 / 2 \pi) \int_{0}^{2 \pi}|f(x)|^{p} d x\right\}^{1 / p}, f \in L_{p}(p \geq 1)$.

A positive sequence $\mathbf{a}:=\left\{a_{n}\right\}$ is called almost monotone decreasing (increasing) if there exists a constant $K:=K(\mathbf{a})$, depending on the sequence a only, such that, for all $n \geq m$, $a_{n} \leq K a_{m}\left(K a_{n} \geq a_{m}\right)$. Such sequences will be denoted by $\mathbf{a} \in$ AMDS and $\mathbf{a} \in$ AMIS, respectively. A sequence which is either AMDS or AMIS is called almost monotone sequence and will be denoted by $\mathbf{a} \in$ AMS.

A sequence $\mathbf{a}:=\left\{a_{n}\right\}$ of nonnegative numbers tending to zero is called a rest bounded variation sequence or briefly $\mathbf{a} \epsilon$ RBVS [5, 6], if it has the property $\sum_{k=m}^{\infty}\left|\Delta a_{k}\right| \leq K(\mathbf{a}) a_{m}$ for all natural numbers $m$, where $K(\mathbf{a})$ is a constant depending upon a only.

A sequence $\mathbf{a}:=\left\{a_{n}\right\}$ of nonnegative numbers is called a head bounded variation sequence or briefly a $\in$ HBVS [6], if it has the property $\sum_{k=0}^{m-1}\left|\Delta a_{k}\right| \leq K(\mathbf{a}) a_{m}$ for all natural numbers $m$, or only for all $m \leq N$ if the sequence a has only a finite number of nonzero terms and the last nonzero term is $a_{N}$.

When we write that a sequence $\left\{a_{n, k}\right\}$ belongs to one of the above classes, it means that it satisfies some of the above conditions with respect to $k=0,1,2, \ldots, n$ for all $n$.

Let $A_{n, k}^{\prime}:=(1 /(k+1)) \sum_{i=0}^{k} a_{n, i}$. If $\left\{A_{n, k}^{\prime}\right\} \in$ AMDS $\left(\left\{A_{n, k}^{\prime}\right\} \in\right.$ AMIS), then we shall say that $\left\{a_{n, k}\right\}$ is an almost monotone decreasing (increasing) mean sequence, or briefly $\left\{a_{n, k}\right\} \in \operatorname{AMDMS}\left(\left\{a_{n, k}\right\} \in\right.$ AMIMS $)$ [7].

Let $A_{n, k}^{\prime \prime}:=(1 /(k+1)) \sum_{i=n-k}^{n} a_{n, i}$. If $\left\{A_{n, k}^{\prime \prime}\right\} \in$ AMDS $\left(\left\{A_{n, k}^{\prime \prime}\right\} \in\right.$ AMIS), then we shall say that $\left\{a_{n, k}\right\}$ is an almost monotone decreasing (increasing) upper mean sequence or briefly $\left\{a_{n, k}\right\} \in$ AMDUMS $\left(\left\{a_{n, k}\right\} \in\right.$ AMIUMS) [8].

Note 2. If NIS (NDS) is the class of nonnegative and nonincreasing (non-decreasing) sequences, then the following inclusion relations are true $[7,8]$ for the above classes of numerical sequences:

NIS $\subset$ RBVS $\subset$ AMDS $\subset$ AMDMS.

NDS $\subset$ HBVS $\subset$ AMIS $\subset$ AMIMS.

Also AMDS $\subset$ AMIUMS and AMIS $\subset$ AMDUMS.
Let $\mathbb{F}$ be an infinite subset of $\mathbb{N}$ and $\mathbb{F}$ as the range of strictly increasing sequence of positive integers, say $\mathbb{F}=$ $\{\lambda(n)\}_{n=1}^{\infty}$. The Cesàro submethod $C_{\lambda}$ is defined as

$$
\left(C_{\lambda} x\right)_{n}=\frac{1}{\lambda(n)} \sum_{k=1}^{\lambda(n)} x_{k}, \quad(n=1,2,3, \ldots)
$$

where $\left\{x_{k}\right\}$ is a sequence of real or complex numbers. Therefore, $C_{\lambda}$-method yields a subsequence of the Cesàro method $C_{1}$, and hence it is regular for any $\lambda . C_{\lambda}$-matrix is obtained by deleting a set of rows from Cesàro matrix. The basic properties of $C_{\lambda}$-method can be found in $[9,10]$.

The notation $[x]$ means the greatest integer contained in $x$.

\section{Known Results}

Let $\sigma_{n}(f), N_{n}(f), R_{n}(f)$ denote the $n$th term of the Cesàro, Nörlund, and Riesz transform of the partial sums of the Fourier series of a $2 \pi$-periodic function $f$, respectively. In 1937, Quade [11] proved that, if $f \in \operatorname{Lip}(\alpha, p)$, then $\| f-$ $\sigma_{n}(f) \|_{p}=O\left(n^{-\alpha}\right)$ for either (i) $p>1$ and $0<\alpha \leq 1$ or (ii) $p=1$ and $0<\alpha<1$. He also showed that, if $p=1=\alpha$, then $\left\|f-\sigma_{n}(f)\right\|_{1}=O\left(n^{-1} \log (n+1)\right)$. These results have been further generalized by various investigators in [5-8, 12-21]. In 2002, Chandra [13] has extended the work of Quade [11] and proved three theorems on the trigonometric approximation using Nörlund and Riesz matrices.

Mittal and Rhoades [22, 23] have initiated the studies of error estimates $E_{n}(f)$ through tfa for the situations in which the summability matrix $T$ does not have monotone rows. Leindler [16] has extended the results of Chandra [13] without the assumption of monotonicity on the generating sequence $\left\{p_{n}\right\}$, while Mittal et al. $[17,18]$ have extended some results of Chandra [13] and Leindler [16] to general matrices. Recently Deger et al. [14] have extended the results of Chandra [13] in view of Armitage and Maddox [9]. Very recently, in [19], the authors of this paper have generalized two theorems of Deger et al. [14], by dropping the monotonicity on the elements of the matrix rows. These results also generalize the results of Leindler [16] to more general $C_{\lambda}$-method.

Szal [8], in 2009, has obtained the same degree of approximation as in theorems proved by Leindler [16] and Chandra [13] for more general class of triangular matrices. He has proved the following theorem for the Nörlund type means.

Theorem A (see [8]). Let $f \in \operatorname{Lip}(\alpha, p)$ and $T \equiv\left(a_{n, k}\right)$ be an infinite lower triangular regular matrix with nonnegative entries and row sums 1 . If one of the conditions,

(i) $p>1,0<\alpha<1$, and $\left\{a_{n, k}\right\} \in$ AMDUMS,

(ii) $p>1,0<\alpha<1,\left\{a_{n, k}\right\} \in$ AMIUMS, and $(n+1) a_{n, n}=$ $O(1)$

(iii) $p>1, \alpha=1$, and $\sum_{k=0}^{n-2}\left|\Delta_{k} A_{n, k}^{\prime \prime}\right|=O\left(n^{-1}\right)$,

(iv) $p=1,0<\alpha<1$, and $\sum_{k=-1}^{n-1}\left|\Delta_{k} a_{n, k}\right|=O\left(n^{-1}\right)$, 
(v) $p=1=\alpha,\left\{(k+1)^{-\beta} a_{n, n-k}\right\} \in H B V S$ for some $\beta>0$, and $(n+1) a_{n, 0}=O(1)$, holds, then

$$
\left\|T_{n}(f)-f\right\|_{p}=O\left(n^{-\alpha}\right)
$$

where $T_{n}(f ; x)=\sum_{k=0}^{n} a_{n, n-k} s_{k}(f ; x)$.

In 2012, Mohapatra and Szal [7] have proved some embedding results on the classes of sequences; few of them are given here in Note 2. Further they proved the following theorem for Riesz type means.

Theorem B (see [7]). Let $f \in \operatorname{Lip}(\alpha, p)$ and $T \equiv\left(a_{n, k}\right)$ be an infinite lower triangular regular matrix with nonnegative entries and row sums 1 . If one of the conditions,

(i) $p>1,0<\alpha<1$, and $\left\{a_{n, k}\right\} \in A M I M S$,

(ii) $p>1,0<\alpha<1,\left\{a_{n, k}\right\} \in A M D M S$, and $(n+1) a_{n, 0}=$ $O(1)$,

(iii) $p>1, \alpha=1$, and $\sum_{k=0}^{n-1}\left|\Delta_{k} A_{n, k}^{\prime}\right|=O\left(n^{-1}\right)$,

(iv) $p=1,0<\alpha<1, \sum_{k=0}^{n-1}\left|\Delta_{k} a_{n, k}\right|=O\left(n^{-1}\right)$, and $(n+$ 1) $a_{n, n}=O(1)$

(v) $p=1,0<\alpha<1,\left(a_{n, k}\right) \in R B V S$, and $(n+1) a_{n, 0}=$ $O(1)$,

(vi) $p=1=\alpha,\left\{(k+1)^{-\beta} a_{n, k}\right\} \in$ HBVS for some $\beta>0$, and $(n+1) a_{n, n}=O(1)$, is maintained, then

$$
\left\|\tau_{n}(f)-f\right\|_{p}=O\left(n^{-\alpha}\right) .
$$

In continuation of Mittal and Singh $[19,20]$, in this paper, we generalize the results of Mohapatra and Szal [7] and Szal [8] using more general $C_{\lambda}$-method to get sharper estimates as mentioned in Remark 6 (given afterwards). Further we show its connections with the existing results as corollaries.

\section{Main Results}

We prove the following theorems:

Theorem 1. Let $f \in \operatorname{Lip}(\alpha, p)$ and $T \equiv\left(a_{n, k}\right)$ be an infinite lower triangular regular matrix with nonnegative entries and row sums 1. If one of the conditions,

(i) $p>1,0<\alpha<1$, and $\left\{a_{n, k}\right\} \in A M I M S$,

(ii) $p>1,0<\alpha<1,\left\{a_{n, k}\right\} \in A M D M S$, and $(\lambda(n)+$ 1) $a_{\lambda(n), 0}=O(1)$

(iii) $p>1, \alpha=1$, and $\sum_{k=0}^{\lambda(n)-2}\left|\Delta_{k} A_{\lambda(n), k}^{\prime}\right|=O\left((\lambda(n))^{-1}\right)$,

(iv) $p=1,0<\alpha<1, \sum_{k=0}^{\lambda(n)-1}\left|\Delta_{k} a_{\lambda(n), k}\right|=O\left((\lambda(n))^{-1}\right)$, and $(\lambda(n)+1) a_{\lambda(n), \lambda(n)}=O(1)$,

(v) $p=1,0<\alpha<1,\left(a_{n, k}\right) \in R B V S$, and $(\lambda(n)+1) a_{\lambda(n), 0}=$ $O(1)$,

(vi) $p=1=\alpha,\left\{(k+1)^{-\beta} a_{\lambda(n), k}\right\} \in$ HBVS for some $\beta>0$, and $(\lambda(n)+1) a_{\lambda(n), \lambda(n)}=O(1)$, is maintained, then

$$
\left\|\tau_{n}^{\lambda}(f)-f\right\|_{p}=O\left((\lambda(n))^{-\alpha}\right),
$$

where $\tau_{n}^{\lambda}(f ; x)=\sum_{k=0}^{\lambda(n)} a_{\lambda(n), k} s_{k}(f ; x), \forall n \geq 0$.
Theorem 2. Let $f \in \operatorname{Lip}(\alpha, p)$ and $T \equiv\left(a_{n, k}\right)$ be defined as in Theorem 1. If one of the conditions,

(i) $p>1,0<\alpha<1$, and $\left\{a_{n, k}\right\} \in$ AMDUMS,

(ii) $p>1,0<\alpha<1,\left\{a_{n, k}\right\} \in$ AMIUMS, and $(\lambda(n)+$ 1) $a_{\lambda(n), \lambda(n)}=O(1)$

(iii) $p>1, \alpha=1$, and $\sum_{k=0}^{\lambda(n)-2}\left|\Delta_{k} A_{\lambda(n), k}^{\prime \prime}\right|=O\left((\lambda(n))^{-1}\right)$,

(iv) $p=1,0<\alpha<1$, and $\sum_{k=-1}^{\lambda(n)-1}\left|\Delta_{k} a_{\lambda(n), k}\right|=$ $O\left((\lambda(n))^{-1}\right)$,

(v) $p=1=\alpha,\left\{(k+1)^{-\beta} a_{\lambda(n), \lambda(n)-k}\right\} \in$ HBVS for some $\beta>0$, and $(\lambda(n)+1) a_{\lambda(n), 0}=O(1)$, holds, then

$$
\left\|T_{n}^{\lambda}(f)-f\right\|_{p}=O\left((\lambda(n))^{-\alpha}\right),
$$

$$
\text { where } T_{n}^{\lambda}(f ; x)=\sum_{k=0}^{\lambda(n)} a_{\lambda(n), \lambda(n)-k} s_{k}(f ; x) .
$$

Corollary 3. If $\lambda(n)=n$, then our Theorems 1 and 2 generalize the results of Mohapatra and Szal [7] and Szal [8], respectively.

Corollary 4. If $a_{n, k}=p_{k} / P_{n}$, then our Theorems 1 and 2 extend very recent results of Değer and Kaya [15].

Corollary 5. In view of Note 2, our Theorems 1 and 2 have been proved with the less stringent conditions than that of results in [20] where the authors have proved a theorem using AMIS, AMDS. Thus our results also generalize the earlier results of Mittal et al. [18, 19], Leindler [16], Chandra [13], and Quade [11].

Remark 6. The estimates in our Theorems 1 and 2 are in terms of $\lambda(n)$ and sharper than the estimates in Mittal et al. [18], Leindler [16], Chandra [13], and Quade [11] as $(\lambda(n))^{-\alpha} \leq n^{-\alpha}$ for $0<\alpha \leq 1$.

\section{Lemmas}

We shall use the following lemmas in the proofs of our Theorems.

Lemma 7 (see [11, p. 538]). If $f \in \operatorname{Lip}(\alpha, p)$, for $p \geq 1,0<$ $\alpha \leq 1$, then, for any positive integer $n, f$ may be approximated in $L_{p}$ by a trigonometric polynomial, $t_{n}$, of order $n$ such that

$$
\left\|f-t_{n}\right\|_{p}=O\left(n^{-\alpha}\right) \text {. }
$$

Lemma 8 (see [11, p. 539]). If $f \in \operatorname{Lip}(\alpha, p)$, for $0<\alpha \leq 1$ and $p>1$, then

$$
\left\|f-s_{n}(f)\right\|_{p}=O\left(n^{-\alpha}\right) .
$$

Lemma 9 (see [11, p. 541]). If $f \in \operatorname{Lip}(\alpha, 1)$, for $0<\alpha<1$, then

$$
\left\|f-\sigma_{n}(f)\right\|_{1}=O\left(n^{-\alpha}\right) .
$$

Lemma 10 (see [11, p. 541, last line]). If $f \in \operatorname{Lip}(1, p)$, for $p>$ 1 , then

$$
\left\|\sigma_{n}(f)-s_{n}(f)\right\|_{p}=O\left(n^{-1}\right), \quad \forall n>0 .
$$


Note 3. We are using sums up to $\lambda(n)$ in the $n$th partial sums $s_{n}$ and $\sigma_{n}$ and writing these sums $s_{n}^{\lambda}$ and $\sigma_{n}^{\lambda}$, respectively, in the above lemmas for our purpose.

Lemma 11. Let $T \equiv\left(a_{n, k}\right)$ be defined as in Theorem 1. If either (i) $\left\{a_{n, k}\right\} \in$ AMIMS or (ii) $\left\{a_{n, k}\right\} \in A M D M S$ and $(\lambda(n)+$ 1) $a_{\lambda(n), 0}=O(1)$, then, for $0<\alpha<1$,

$$
\sum_{k=0}^{\lambda(n)} a_{\lambda(n), k}(k+1)^{-\alpha}=O\left((\lambda(n))^{-\alpha}\right) .
$$

Proof. Let $r:=[\lambda(n) / 2]$. Then

$$
\begin{aligned}
\sum_{k=0}^{\lambda(n)} a_{\lambda(n), k}(k+1)^{-\alpha} & =\left(\sum_{k=0}^{r}+\sum_{k=r+1}^{\lambda(n)}\right) a_{\lambda(n), k}(k+1)^{-\alpha} \\
& \leq \sum_{k=0}^{r} a_{\lambda(n), k}(k+1)^{-\alpha}+(r+1)^{-\alpha} .
\end{aligned}
$$

By Abel's transformation and using the Lagrange Mean Value Theorem to the function $f(x)=x^{-\alpha}, 0<\alpha<1$ on the interval $(k+1, k+2)$, we get

$$
\begin{aligned}
& \sum_{k=0}^{r} a_{\lambda(n), k}(k+1)^{-\alpha} \\
& \leq \sum_{k=0}^{r-1}\left\{(k+1)^{-\alpha}-(k+2)^{-\alpha}\right\} \sum_{i=0}^{k} a_{\lambda(n), i}+(r+1)^{-\alpha} \\
& \leq \sum_{k=0}^{r-1} \frac{(k+1)^{-\alpha}-(k+2)^{-\alpha}}{(k+1)^{-\alpha}(k+2)^{-\alpha}}(k+1) A_{\lambda(n), k}^{\prime} \\
& \quad+(r+1)^{-\alpha} \leq \sum_{k=0}^{r-1} \frac{\alpha}{(k+2)^{\alpha}} A_{\lambda(n), k}^{\prime}+(r+1)^{-\alpha} .
\end{aligned}
$$

(i) If $\left\{a_{n, k}\right\} \in$ AMIMS, that is, $\left\{A_{n, k}^{\prime}\right\} \in$ AMIS, then

$$
\begin{aligned}
\sum_{k=0}^{\lambda(n)} a_{\lambda(n), k}(k+1)^{-\alpha}= & O\left(\sum_{k=0}^{r-1} \frac{\alpha}{(k+2)^{\alpha}} A_{\lambda(n), k}^{\prime}\right) \\
& +O\left((r+1)^{-\alpha}\right) \\
= & O\left(A_{\lambda(n), r}^{\prime} \sum_{k=0}^{r-1} \frac{\alpha}{(k+2)^{\alpha}}\right) \\
& +O\left((r+1)^{-\alpha}\right) \\
= & O\left((r+1)^{-\alpha} \sum_{k=0}^{r} a_{\lambda(n), k}\right) \\
& +O\left((r+1)^{-\alpha}\right)=O\left((r+1)^{-\alpha}\right) \\
= & O\left((\lambda(n))^{-\alpha}\right) .
\end{aligned}
$$

(ii) If $\left\{a_{n, k}\right\} \in$ AMDMS, that is, $\left\{A_{n, k}^{\prime}\right\} \in$ AMDS, and $(\lambda(n)+$ 1) $a_{\lambda(n), 0}=O(1)$, then, as mentioned above, the proof of this case runs parallel to that of case (i).
Lemma 12. Let $t_{n}$ be a trigonometric polynomial as in Lemma 7. If $\left\{(k+1)^{-\beta} a_{\lambda(n), k}\right\} \in H B V S$ for some $\beta>0$ and $(\lambda(n)+1) a_{\lambda(n), \lambda(n)}=O(1)$, then

$$
\left\|\tau_{n}^{\lambda}(f)-\sum_{k=0}^{\lambda(n)} a_{\lambda(n), k} t_{k}\right\|_{1}=O\left((\lambda(n))^{-1}\right) .
$$

Proof. If $s_{k}\left(t_{\lambda(n)} ; x\right)$ denotes the partial sum of the first $(k+1)$ terms of the Fourier series of $t_{\lambda(n)}$ (as in [13, p. 14 \& 21]) at $x$, then for $k \leq \lambda(n)$

$$
\begin{aligned}
s_{k}\left(t_{\lambda(n)} ; x\right) & =t_{k}(x), \\
s_{k}(f ; x)-t_{k}(x) & =s_{k}\left(f-t_{\lambda(n)} ; x\right) .
\end{aligned}
$$

Thus

$$
\tau_{n}^{\lambda}(f)-\sum_{k=0}^{\lambda(n)} a_{\lambda(n), k} t_{k}(x)=\sum_{k=0}^{\lambda(n)} a_{\lambda(n), k} s_{k}\left(f-t_{\lambda(n)} ; x\right),
$$

where

$$
\begin{aligned}
& s_{k}\left(f-t_{\lambda(n)} ; x\right)=\frac{1}{\pi} \int_{0}^{2 \pi}\left\{f(x+u)-t_{\lambda(n)}(x+u)\right\} \\
& . \frac{\sin (k+(1 / 2)) u}{2 \sin (u / 2)} d u .
\end{aligned}
$$

Using the generalized Minkowski inequality [24, p. 37], we get

$$
\begin{aligned}
& \left\|\tau_{n}^{\lambda}(f)-\sum_{k=0}^{\lambda(n)} a_{\lambda(n), k} t_{k}\right\|_{1}=\frac{1}{2 \pi^{2}} \\
& \cdot \int_{0}^{2 \pi} \mid \int_{0}^{2 \pi}\left[f(x+u)-t_{\lambda(n)}(x+u)\right] \\
& \cdot K_{n}^{\lambda}(u) d u\left|d x \leq \frac{1}{2 \pi^{2}} \int_{0}^{2 \pi}\right| K_{n}^{\lambda}(u) \mid \\
& \cdot\left(\int_{0}^{2 \pi}\left|f(x+u)-t_{\lambda(n)}(x+u)\right| d x\right) d u \\
& =\frac{1}{2 \pi^{2}} \int_{0}^{2 \pi}\left|K_{n}^{\lambda}(u)\right| d u \int_{0}^{2 \pi}\left|f(x)-t_{\lambda(n)}(x)\right| d x \\
& =\frac{2}{\pi}\left\|f-t_{\lambda(n)}\right\|_{1} \int_{0}^{\pi}\left|K_{n}^{\lambda}(u)\right| d u=\frac{2}{\pi}\left\|f-t_{\lambda(n)}\right\|_{1} \\
& \cdot\left(\int_{0}^{\pi / \lambda(n)}\left|K_{n}^{\lambda}(u)\right| d u+\int_{\pi / \lambda(n)}^{\pi}\left|K_{n}^{\lambda}(u)\right| d u\right) \\
& =\frac{2}{\pi}\left\|f-t_{\lambda(n)}\right\|_{1}\left(I_{1}+I_{2}\right), \text { say, }
\end{aligned}
$$

where $K_{n}^{\lambda}(u)=\sum_{k=0}^{\lambda(n)} a_{\lambda(n), k}(\sin (k+(1 / 2)) u / 2 \sin (u / 2))$.

Now using $|\sin k t| \leq k|\sin t|$, we have $I_{1}=$ $O\left(\int_{0}^{\pi / \lambda(n)}(\lambda(n)+1) \sum_{k=0}^{\lambda(n)} a_{\lambda(n), k} d u\right)=O(1)$. 
If $\left\{(k+1)^{-\beta} a_{\lambda(n), k}\right\} \in$ HBVS for some $\beta>0$, then $\{(k+$ $\left.1)^{-\beta} a_{\lambda(n), k}\right\} \in$ AMIS as HBVS $\subset$ AMIS. This further implies that $\left\{a_{\lambda(n), k}\right\} \in$ AMIS, since, for $0 \leq k \leq m \leq \lambda(n)$,

$$
a_{\lambda(n), k} \leq a_{\lambda(n), k}\left(\frac{m+1}{k+1}\right)^{\beta} \leq K(\mathbf{a}) a_{\lambda(n), m} .
$$

Using $\left\{a_{\lambda(n), k}\right\} \in$ AMIS, $(\sin u)^{-1} \leq(2 u / \pi)^{-1}(0 \leq u \leq \pi / 2)$, and $(\lambda(n)+1) a_{\lambda(n), \lambda(n)}=O(1)$,

$$
\begin{aligned}
I_{2} & =O\left(\int_{\pi / \lambda(n)}^{\pi}\left|a_{\lambda(n), \lambda(n)} \sum_{k=0}^{\lambda(n)} \frac{\sin (k+(1 / 2)) u}{2 \sin (u / 2)}\right| d u\right) \\
& =O\left(a_{\lambda(n), \lambda(n)}\right) \int_{\pi / \lambda(n)}^{\pi} u^{-2} d u=O(1) .
\end{aligned}
$$

Putting $I_{1}, I_{2}$ in (22) and using Lemma 7, we get

$$
\begin{aligned}
& \left\|\tau_{n}^{\lambda}(f)-\sum_{k=0}^{\lambda(n)} a_{\lambda(n), k} t_{k}\right\|_{1}=O\left(\left\|f-t_{\lambda(n)}\right\|_{1}\right) \\
& =O\left((\lambda(n))^{-1}\right) .
\end{aligned}
$$

This completes the proof of Lemma 12.

Lemma 13. Let $T \equiv\left(a_{n, k}\right)$ be defined as in Theorem 1. If either (i) $\left\{a_{n, k}\right\} \in A M D U M S$ or (ii) $\left\{a_{n, k}\right\} \in A M I U M S$ and $(\lambda(n)+$ 1) $a_{\lambda(n), \lambda(n)}=O(1)$. Then for $0<\alpha<1$,

$$
\sum_{k=0}^{\lambda(n)} a_{\lambda(n), \lambda(n)-k}(k+1)^{-\alpha}=O\left((\lambda(n))^{-\alpha}\right) .
$$

Lemma 14. Let $t_{n}$ be a trigonometric polynomial as in Lemma 7. If $\left\{(k+1)^{-\beta} a_{\lambda(n), \lambda(n)-k}\right\} \in H B V S$ for some $\beta>0$ and $(\lambda(n)+1) a_{\lambda(n), 0}=O(1)$, then

$$
\left\|T_{n}^{\lambda}(f)-\sum_{k=0}^{\lambda(n)} a_{\lambda(n), \lambda(n)-k} t_{k}\right\|_{1}=O\left((\lambda(n))^{-1}\right) .
$$

We omit the proofs of Lemmas 13 and 14 as they are similar to that of Lemmas 11 and 12 , respectively.

\section{Proofs of Main Results}

\subsection{Proof of the Theorem 1}

Cases (i) and (ii). Since $\tau_{n}^{\lambda}(f ; x)-f(x)=\sum_{k=0}^{\lambda(n)} a_{\lambda(n), k}\left(s_{k}(f\right.$; $x)-f(x)$ ), thus, using Lemmas 8 and 11, we have

$$
\begin{aligned}
\left\|\tau_{n}^{\lambda}(f)-f\right\|_{p} & \leq \sum_{k=0}^{\lambda(n)} a_{\lambda(n), k}\left\|s_{k}(f)-f\right\|_{p} \\
& =O\left(\sum_{k=0}^{\lambda(n)} a_{\lambda(n), k}(k+1)^{-\alpha}\right) \\
& =O\left((\lambda(n))^{-\alpha}\right) .
\end{aligned}
$$

This completes the proofs of (i) and (ii).
Case (iii). In view of Note 3, we use $s_{\lambda(n)}$ as $s_{n}^{\lambda}$ and then, using Abel's transformation twice,

$$
\begin{aligned}
& \tau_{n}^{\lambda}(f ; x)-f(x) \\
& =\sum_{k=0}^{\lambda(n)-1}\left[s_{k}(f ; x)-s_{k+1}(f ; x)\right] \sum_{i=0}^{k} a_{\lambda(n), i} \\
& +\left[s_{n}^{\lambda}(f ; x)-f(x)\right] \\
& =s_{n}^{\lambda}(f ; x)-f(x) \\
& -\sum_{k=0}^{\lambda(n)-1}(k+1) u_{k+1}(f ; x) A_{\lambda(n), k}^{\prime} \\
& =s_{n}^{\lambda}(f ; x)-f(x) \\
& -\sum_{k=0}^{\lambda(n)-2}\left(A_{\lambda(n), k}^{\prime}-A_{\lambda(n), k+1}^{\prime}\right) \sum_{i=0}^{k}(i+1) u_{i+1}(f ; x) \\
& -A_{\lambda(n), \lambda(n)-1}^{\prime} \sum_{i=0}^{\lambda(n)-1}(i+1) u_{i+1}(f ; x) \\
& =s_{n}^{\lambda}(f ; x)-f(x) \\
& -\sum_{k=0}^{\lambda(n)-2}\left(A_{\lambda(n), k}^{\prime}-A_{\lambda(n), k+1}^{\prime}\right) \sum_{i=1}^{k+1} i u_{i}(f ; x) \\
& -(\lambda(n))^{-1} \sum_{k=0}^{\lambda(n)} a_{\lambda(n), k} \sum_{i=1}^{\lambda(n)} i u_{i}(f ; x) .
\end{aligned}
$$

Hence by Minkowski's inequality, we get

$$
\begin{aligned}
\left\|\tau_{n}^{\lambda}(f)-f\right\|_{p} \leq & \left\|s_{n}^{\lambda}(f)-f\right\|_{p} \\
& +\sum_{k=0}^{\lambda(n)-2}\left\|\sum_{i=1}^{k+1} i u_{i}(f ; x)\right\|_{p}\left|\Delta_{k} A_{\lambda(n), k}^{\prime}\right| \\
& +(\lambda(n))^{-1}\left\|\sum_{i=1}^{\lambda(n)} i u_{i}(f)\right\|_{p} .
\end{aligned}
$$

Since

$$
s_{n}^{\lambda}(f)-\sigma_{n}^{\lambda}(f)=\frac{1}{\lambda(n)+1} \sum_{i=1}^{\lambda(n)} i u_{i}(f ; x),
$$

thus by Lemma 10

$$
\left\|\sum_{i=1}^{\lambda(n)} i u_{i}\right\|_{p}=(\lambda(n)+1)\left\|\sigma_{n}^{\lambda}(f)-s_{n}^{\lambda}(f)\right\|_{p}=O(1) .
$$

If $\sum_{k=0}^{\lambda(n)-2}\left|\Delta_{k} A_{\lambda(n), k}^{\prime}\right|=O\left(\lambda(n)^{-1}\right)$, then

$$
\begin{aligned}
\left\|\tau_{n}^{\lambda}(f)-f\right\|_{p} & =O\left(\lambda(n)^{-1}\right)+O(1) \sum_{k=0}^{\lambda(n)-2}\left|\Delta_{k} A_{\lambda(n), k}^{\prime}\right| \\
& =O\left((\lambda(n))^{-1}\right),
\end{aligned}
$$

in view of (30), (32), and Lemma 8. 
Cases (iv) and (v). Using Abel's transformation,

$$
\begin{aligned}
\tau_{n}^{\lambda}(f ; x)-f(x) \\
=\sum_{k=0}^{\lambda(n)-1}\left(\Delta_{k} a_{\lambda(n), k}\right) \sum_{i=0}^{k}\left[s_{i}(f ; x)-f(x)\right] \\
\quad+a_{\lambda(n), \lambda(n)} \sum_{i=0}^{\lambda(n)}\left[s_{i}(f ; x)-f(x)\right] \\
=\sum_{k=0}^{\lambda(n)-1}\left(\Delta_{k} a_{\lambda(n), k}\right)(k+1)\left[\sigma_{k}(f ; x)-f(x)\right] \\
\quad+a_{\lambda(n), \lambda(n)}(\lambda(n)+1)\left[\sigma_{n}^{\lambda}(f ; x)-f(x)\right] .
\end{aligned}
$$

Using Lemma 9, we get

$$
\begin{aligned}
& \left\|\tau_{n}^{\lambda}(f)-f\right\|_{1} \\
& \leq \sum_{k=0}^{\lambda(n)-1}(k+1)\left|\Delta_{k} a_{\lambda(n), k}\right|\left\|\sigma_{k}(f)-f\right\|_{1} \\
& \quad+a_{\lambda(n), \lambda(n)}(\lambda(n)+1)\left\|\sigma_{n}^{\lambda}(f)-f\right\|_{1} \\
& \leq \sum_{k=0}^{\lambda(n)-1}\left|\Delta_{k} a_{\lambda(n), k}\right|(k+1)^{1-\alpha} \\
& \quad+a_{\lambda(n), \lambda(n)}(\lambda(n)+1)^{1-\alpha} \\
& \leq(\lambda(n)+1)^{1-\alpha}\left(\sum_{k=0}^{\lambda(n)-1}\left|\Delta_{k} a_{\lambda(n), k}\right|+a_{\lambda(n), \lambda(n)}\right) .
\end{aligned}
$$

Using conditions $\sum_{k=0}^{\lambda(n)-1}\left|\Delta_{k} a_{\lambda(n), k}\right|=O\left((\lambda(n))^{-1}\right)$ and $(\lambda(n)+1) a_{\lambda(n), \lambda(n)}=O(1)$, we get

$$
\left\|\tau_{n}^{\lambda}(f)-f\right\|_{1}=O\left((\lambda(n))^{-\alpha}\right) .
$$

This completes the proof of case (iv).

Further, if $\left(a_{n, k}\right) \in$ RBVS, then we have

$$
\begin{aligned}
\sum_{k=0}^{\lambda(n)-1}\left|\Delta_{k} a_{\lambda(n), k}\right| & =\sum_{k=0}^{\lambda(n)-1}\left|a_{\lambda(n), k}-a_{\lambda(n), k+1}\right| \\
& \leq K(\mathbf{a}) a_{\lambda(n), 0} .
\end{aligned}
$$

Therefore from (35), (37), and $\left(a_{n, k}\right) \in$ AMDS (as RBVS $\subset$ AMDS), we get

$$
\begin{aligned}
& \left\|\tau_{n}^{\lambda}(f)-f\right\|_{1} \\
& \quad=O\left((\lambda(n)+1)^{1-\alpha}\right)\left(a_{\lambda(n), 0}+a_{\lambda(n), \lambda(n)}\right) \\
& \quad=O\left((\lambda(n)+1)^{1-\alpha}\right) a_{\lambda(n), 0}=O\left((\lambda(n))^{-\alpha}\right),
\end{aligned}
$$

since $(\lambda(n)+1) a_{\lambda(n), 0}=O(1)$. This completes the proof of case $(\mathrm{v})$.
Case (vi). By Lemmas 7 and 12, we have

$$
\begin{aligned}
\left\|\tau_{n}^{\lambda}(f)-f\right\|_{1} \leq & \left\|\tau_{n}^{\lambda}(f)-\sum_{k=0}^{\lambda(n)} a_{\lambda(n), k} t_{k}\right\|_{1} \\
& +\left\|\sum_{k=0}^{\lambda(n)} a_{\lambda(n), k} t_{k}-f\right\|_{1} \\
\left\|\tau_{n}^{\lambda}(f)-f\right\|_{1}= & O\left((\lambda(n))^{-1}\right) \\
& +O\left(\sum_{k=0}^{\lambda(n)} a_{\lambda(n), k}\left\|t_{k}-f\right\|_{1}\right) \\
= & O\left((\lambda(n))^{-1}\right) \\
& +O\left(\sum_{k=0}^{\lambda(n)} a_{\lambda(n), k}(k+1)^{-1}\right) .
\end{aligned}
$$

Using Abel's transformation, we get

$$
\begin{aligned}
& \sum_{k=0}^{\lambda(n)} a_{\lambda(n), k}(k+1)^{-1}=\sum_{k=0}^{\lambda(n)-1}\left|\frac{a_{\lambda(n), k}}{(k+1)^{\beta}}-\frac{a_{\lambda(n), k+1}}{(k+2)^{\beta}}\right| \\
& \cdot \sum_{i=0}^{k}(i+1)^{\beta-1}+\frac{a_{\lambda(n), \lambda(n)}}{(\lambda(n)+1)^{\beta}} \sum_{i=0}^{\lambda(n)}(i+1)^{\beta-1} \\
& =O\left((\lambda(n)+1)^{\beta} \sum_{k=0}^{\lambda(n)-1}\left|\frac{a_{\lambda(n), k}}{(k+1)^{\beta}}-\frac{a_{\lambda(n), k+1}}{(k+2)^{\beta}}\right|\right. \\
& \left.+a_{\lambda(n), \lambda(n)}\right)=O\left((\lambda(n))^{-1}\right),
\end{aligned}
$$

since $\left\{(k+1)^{-\beta} a_{\lambda(n), k}\right\} \in \operatorname{HBVS}$ and $(\lambda(n)+1) a_{\lambda(n), \lambda(n)}=O(1)$. This completes the proof of case (vi) and hence the proof of Theorem 1.

5.2. Proof of the Theorem 2. We omit the details as the proof is similar to that of Theorem 1 . We use Lemmas 13 and 14 instead of Lemmas 11 and 12.

\section{New Contribution}

In classical Fourier analysis many results on tfa of functions in certain function spaces have been established using monotonicity condition on the elements of summability matrices. These results can be generalized in two possible directions. One is to use more general method and the other is to weaken the monotonicity condition. To achieve the latter aim, various generalizations of the monotonicity condition such as semimonotonicity, quasimonotonicity, AMIS, AMDS, HBVS, and RBVS were suggested by many authors from time to time. Weakening the monotonicity condition will enhance the quality of a digital filter in view of Note 1.

Motivated by these ideas we improve the results of Mohapatra and Szal [7] and Szal [8] using Cesàro submethod which in turn generalize various known results in the light of 
recent developments and in view of Note 2. We also note that we get sharper estimates than that of $[7,8]$ as $(\lambda(n))^{-\alpha} \leq n^{-\alpha}$ for $0<\alpha \leq 1$. A few more applications of sharper estimates and tfa can be seen in [18, p. 4484].

Remark 15. The study of degree of approximation of functions in other function spaces such as $\operatorname{Lip}(\xi(t), p)$, weighted Lipschitz, Hölder, and Zygmund classes may be the future interest of few investigators in this direction.

\section{Conflict of Interests}

The authors declare that there is no conflict of interests regarding the publication of this paper.

\section{Acknowledgment}

The second author is thankful to Ministry of Human Resource Development, India, for financial support to carry out this research work.

\section{References}

[1] E. Z. Psarakis and G. V. Moustakides, "An $\mathrm{L}_{2}$-based method for the design of 1-D zero phase FIR digital filters," IEEE Transactions on Circuits and Systems I: Fundamental Theory and Applications, vol. 44, no. 7, pp. 591-601, 1997.

[2] W. K. Chen, Mathematics for Circuits and Filters, CRC Press, 2000.

[3] J. G. Proakis, Digital Communications, McGraw-Hill, New York, NY, USA, 1995.

[4] A. Zygmund, Trigonometric Series. Volume I, II, Cambridge Mathematical Library, Cambridge University Press, Cambridge, UK, 1988, reprint of the 1979 edition.

[5] L. Leindler, "On the uniform convergence and boundedness of a certain class of sine series," Analysis Mathematica, vol. 27, no. 4, pp. 279-285, 2001.

[6] L. Leindler, "On the degree of approximation of continuous functions," Acta Mathematica Hungarica, vol. 104, no. 1-2, pp. 105-113, 2004.

[7] R. N. Mohapatra and B. Szal, "On trigonometric approximation offunctions in the $L_{p}$-norm," http://arxiv.org/abs/1205.5869.

[8] B. Szal, "Trigonometric approximation by Nörlund type mean in $L_{p}$-norm," Commentationes Mathematicae Universitatis Carolinae, vol. 50, no. 4, pp. 575-589, 2009.

[9] D. H. Armitage and I. J. Maddox, "A new type of Cesàro mean," Analysis, vol. 9, no. 1-2, pp. 195-204, 1989.

[10] J. A. Osikiewicz, "Equivalence results for Cesàro submethods," Analysis, vol. 20, no. 1, pp. 35-43, 2000.

[11] E. S. Quade, "Trigonometric approximation in the mean," Duke Mathematical Journal, vol. 3, no. 3, pp. 529-543, 1937.

[12] P. Chandra, "A note on degree of approximation by Nörlund and Riesz operators," Matematicki Vesnik, vol. 42, no. 1, pp. 9-10, 1990.

[13] P. Chandra, "Trigonometric approximation of functions in $L_{p^{-}}$ norm," Journal of Mathematical Analysis and Applications, vol. 275, no. 1, pp. 13-26, 2002.

[14] U. Deger, I. Dagadur, and M. Küçükaslan, "Approximation by trigonometric polynomials to functions in $L_{p}$-norm," Proceedings of the Jangjeon Mathematical Society, vol. 15, no. 2, pp. 203213, 2012.
[15] U. Deǧer and M. Kaya, "On the approximation by Cesàro submethod," Palestine Journal of Mathematics, vol. 4, no. 1, pp. 44$56,2015$.

[16] L. Leindler, “Trigonometric approximation in $L_{p}$-norm," Journal of Mathematical Analysis and Applications, vol. 302, no. 1, pp. 129-136, 2005.

[17] M. L. Mittal, B. E. Rhoades, V. N. Mishra, and U. Singh, "Using infinite matrices to approximate functions of class $\operatorname{Lip}(\alpha, \mathrm{p})$ using trigonometric polynomials," Journal of Mathematical Analysis and Applications, vol. 326, no. 1, pp. 667-676, 2007.

[18] M. L. Mittal, B. E. Rhoades, S. Sonker, and U. Singh, "Approximation of signals of class $\operatorname{Lip}(\alpha, p)$ by linear operators," Applied Mathematics and Computation, vol. 217, no. 9, pp. 4483-4489, 2011.

[19] M. L. Mittal and M. V. Singh, "Approximation of signals (functions) by trigonometric polynomials in $L_{p}$-norm," International Journal of Mathematics and Mathematical Sciences, vol. 2014, Article ID 267383, 6 pages, 2014.

[20] M. L. Mittal and M. V. Singh, "Approximation of functions of class $\operatorname{Lip}(\alpha, p)$ in $L_{p}$-norm," in Mathematical Analysis and its Applications: Roorkee, India, December 2014, Springer Proceedings in Mathematics \& Statistics, pp. 109-120, Springer, Berkeley, Calif, USA, 2015.

[21] R. N. Mohapatra and D. C. Russell, "Some direct and inverse theorems in approximation of functions," Australian Mathematical Society. Series A. Pure Mathematics and Statistics, vol. 34, no. 2, pp. 143-154, 1983.

[22] M. L. Mittal and B. E. Rhoades, "On the degree of approximation of continuous functions by using linear operators on their Fourier series," International Journal of Mathematics, Game Theory, and Algebra, vol. 9, no. 4, pp. 259-267, 1999.

[23] M. L. Mittal and B. E. Rhoades, "Degree of approximation to functions in a normed space," Journal of Computational Analysis and Applications, vol. 2, no. 1, pp. 1-10, 2000.

[24] C. K. Chui, An Introduction to Wavelets, vol. 1 of Wavelet Analysis and Its Applications, Academic Press, New York, NY, USA, 1992 


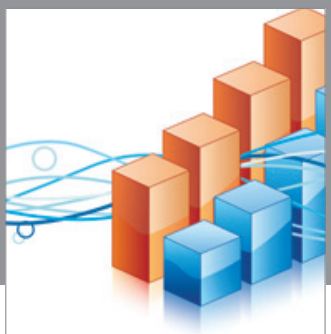

Advances in

Operations Research

vatem alat4

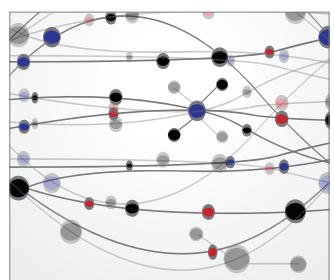

\section{The Scientific} World Journal
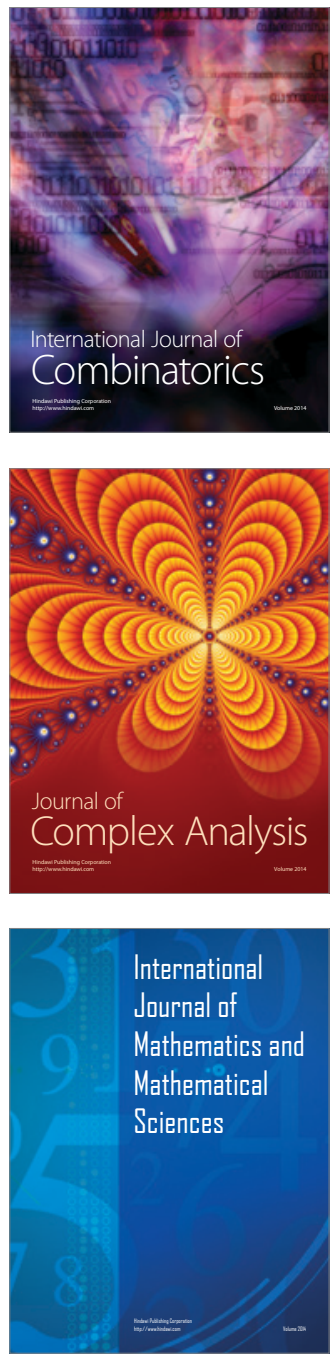
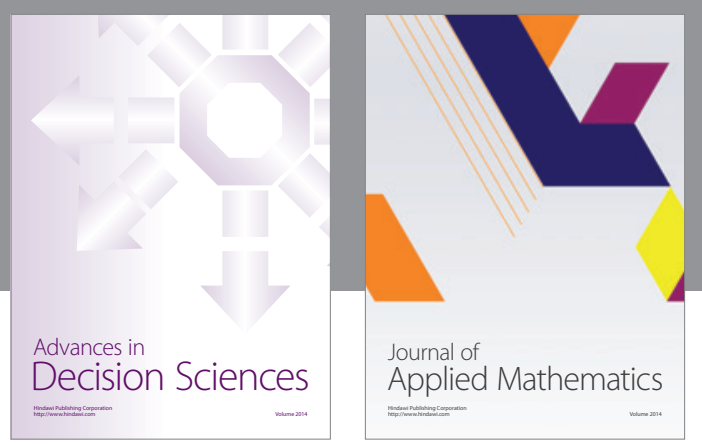

Algebra

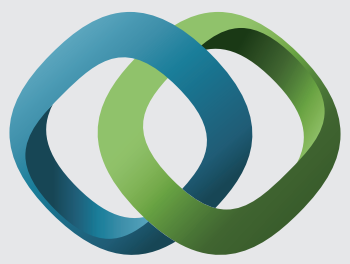

\section{Hindawi}

Submit your manuscripts at

http://www.hindawi.com
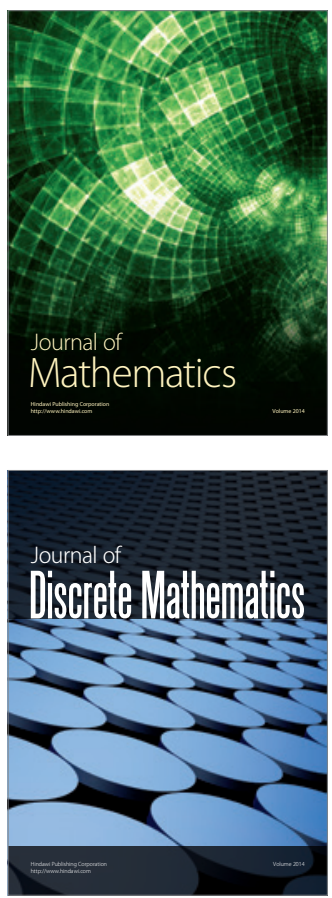

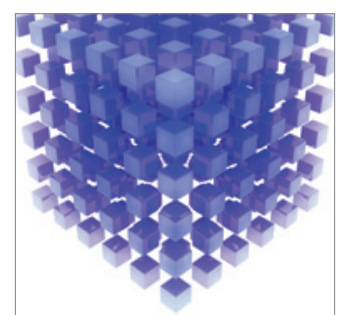

Mathematical Problems in Engineering
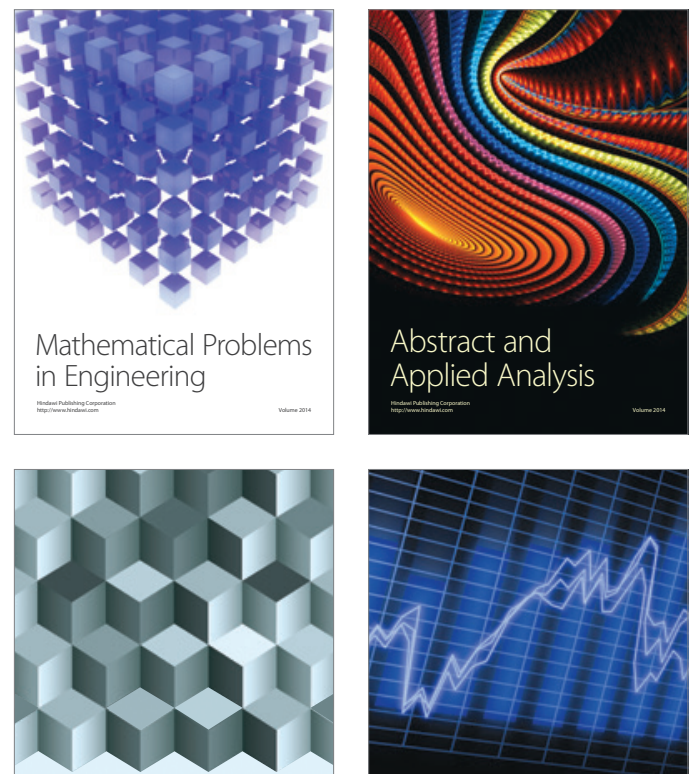

Journal of

Function Spaces

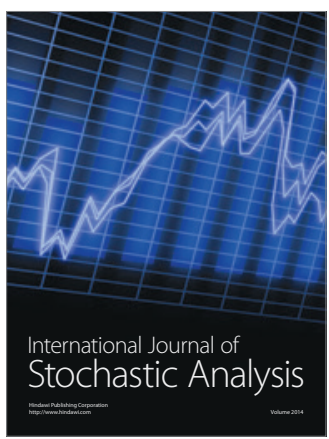

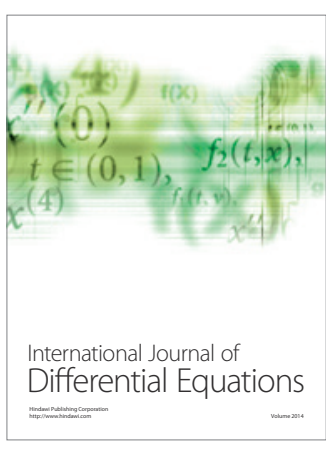
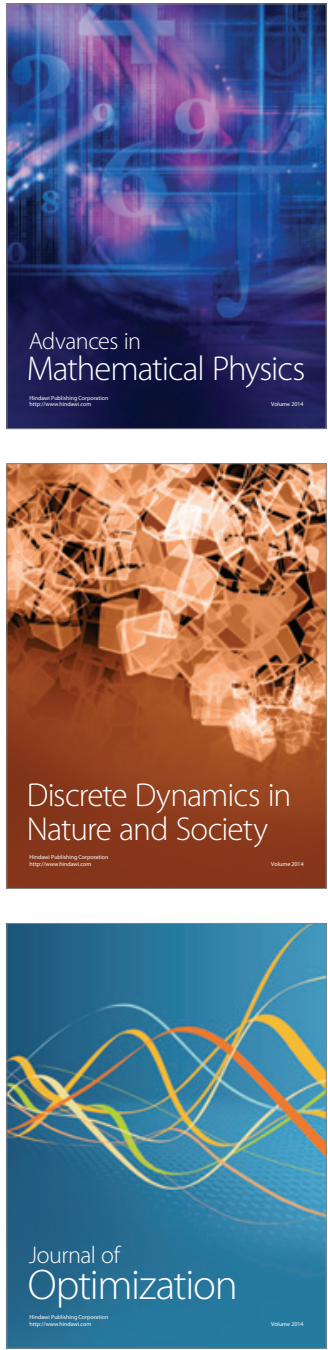